\title{
Short-term memory for visual and auditory stimuli in pigeons
}

\author{
PHILIPP J. KRAEMER and WILLIAM A. ROBERTS \\ University of Western Ontario, London, Ontario, Canada
}

\begin{abstract}
Separate groups of pigeons were trained to perform symbolic delayed matching to sample with auditory and visual sample stimuli. For unimals in the auditory group, ambient tones that varied in frequency served as sample stimuli; for animals in the visual group, ambient red and green lights served as sample stimuli. In both cases, the sample stimuli were mapped onto the yellow and blue comparison stimuli presented on left and right pecking keys. In Experiments 1 and 2 , it was found that visual and auditory delayed matching were affected in the same ways by several temporal variables: delay, length of exposure to the sample stimulus, and intertrial interval. In Experiments 3, 4A, and 4B, a houselight presented during the delay interval strongly interfered with retention in both visual and auditory groups, but white noise presented during the delay had little effect in either group. These results seem to be more in line with a prospective memory model, in which visual and auditory sample stimuli are coded into the same instructional memories, than with a model based on concepts of retrospective memory and modality specificity.
\end{abstract}

Studies of short-term memory (STM) in pigeons typically have made use of delayed simultaneous or successive matching-to-sample procedures, in which the sample and comparison stimuli are visual displays presented on circular keys. When an identity relationship is used between sample and comparison stimuli, trials involve the presentation of the same visual display, commonly a colored field or a black-and-white geometric pattern, as the sample and matching comparison stimuli (e.g., Blough, 1959; Nelson \& Wasserman, 1978; Roberts, 1972). In symbolic delayed matching procedures, arbitrary relationships may be mapped between different sample and comparison stimuli; thus, a red sample stimulus may inform an animal that pecking a comparison key showing vertical black and white stripes will yield reinforcement (G. B. Peterson, Wheeler, \& Armstrong, 1978; Roitblat, 1980). In some symbolic delayed matching experiments, visual comparison stimuli have been mapped onto multimodal sample stimuli, such as samples of food and no food, number of pecks on a lit sample key, or duration of exposure to the houselight (D. S. Grant, 1982a, 1982b; Maki, Moe, \& Bierley, 1977; Spetch \& Wilkie, 1983). A visual component was present in the sample stimulus in each of these cases of delayed symbolic matching to multimodal sample stimuli.

Few experiments have been done with animals to examine possible differences beween auditory and visual

Support for this research was provided by Grant A7894 from the Natural Sciences and Engineering Research Council of Canada. Requests for reprints should be sent to William $A$. Roberts, Department of Psychology, University of Western Ontario, London, Ontario, Canada N6A 5C2.
STM. Experiments that have directly compared auditory and visual memory have shown that rats retain auditory memories longer than visual memories (Cohen, Escott, \& Ricciardi, 1984; Wallace, Steinert, Scobie, \& Spear, 1978). Although most work done with birds and primates has used visual cues, the use of sound stimuli has dominated research with dolphins. Well known for their auditory acuity, dolphins have shown excellent STM for single sample sounds or lists of sounds (Herman \& Gordon, 1974; Thompson \& Herman, 1977). Furthermore, dolphins perform well in auditory delayed matching when sample and comparison stimuli are either identical or symbolically related (Herman \& Thompson, 1982). In contrast to their success with auditory stimuli, dolphins have difficulty learning to match to sample with visual stimuli and would not be expected to show persistent STM for visual events (Herman, 1980).

Research done with human subjects has provided substantial evidence that verbal items are better remembered over short periods when presented in the auditory modality than when presented in the visual modality. In experiments using the Peterson technique (L. R. Peterson \& M. J. Peterson, 1959), strings of consonants presented in the auditory modality are better remembered than those presented in the visual modality up to a retention interval of about $6 \mathrm{sec}$ (Cooley \& McNulty, 1967; K. W. Grant \& McCormack, 1969). When memory for lists of items has been examined, the modality effect typically has taken the form of an interaction, in which the final items but not the earlier items of a list are better remembered following auditory than following visual presentation (Conrad \& Hull, 1968; Murdock \& Walker, 1969). This effect has been revealed under a variety of memory testing procedures, including free 
recall, serial recall, and recognition tests (Murdock, 1968; Watkins, Watkins, \& Crowder, 1974).

There appear to be no experiments that directly compare pigeon STM for stimuli presented in one sensory modality with that for stimuli presented in another sensory modality. In the experiments presented here, we examined pigeons' STM for visual and auditory stimuli. Separate groups of visual and auditory birds either saw ambient houselights varying in color or heard ambient tones varying in frequency as sample stimuli. For both groups, these sample stimuli were symbolically mapped onto the same colored comparison stimuli presented on left and right keys. In this way, discriminability of the comparison stimuli was held constant, and differences in performance could be attributed to differences in the sample stimuli. In these experiments, we were concerned with the possibility that memory for information from these two modalities might be qualitatively different in some ways. If auditory and visual memories have different attributes, we might find that they are differently affected by experimental variables. In the following experiments, we examined the effects of a number of variables on delayed visual and auditory matching; these variables included delay, sample exposure time, intertrial interval (ITI), interstimulus interval (ISI), and the presentation of visual and auditory stimulation between sample and comparison stimuli. The effects of these variables on visual STM in pigeons are well known, but their effects on auditory STM are unknown. If it was found that some of these variables had no effect on performance in auditory STM, or that they affected auditory STM differently from visual STM, we could begin to argue for memory systems with different properties.

The suggestion that auditory and visual STM might be qualitatively different implies the possibility that memories of visual and auditory stimuli are stored in different parts of the brain and that they differ in the informational components that are retained. Such a position assumes that STM in pigeons is retrospective and preserves some of the characteristics of the sample stimulus. An alternative possibility is that STM is instructional or prospective (D. S. Grant, 1981; Honig \& Thompson, 1982; Roitblat, 1980). In this case, both auditory and visual sample stimuli would be rapidly coded into instructions about which comparison stimulus to peck at the end of the delay, and the information held in memory during the delay would not differ between modalities of sample stimulus presentation. The prospective memory theoretical position leads to the expectation that manipulations of experimental variables should have little differential effect on delayed matching with auditory and visual sample stimuli.

\section{EXPERIMENT 1}

Two variables that strongly affect performance in delayed matching with visual cues are sample presenta- tion time and delay between sample and comparison stimuli. Accuracy has been found to be directly related to length of exposure to the sample stimulus and to be inversely related to length of the delay (Roberts, 1972; Roberts \& Grant, 1974). In this initial experiment, the effects of presentation time and delay on visual and auditory delayed matching were examined within a $2 \times 3 \times 4$ factorial design. Delayed matching was studied in groups of auditory and visual birds, with presentation time set at values of 1,4 , and $8 \mathrm{sec}$ and delay set at values of $0, .5,1$, and $3 \mathrm{sec}$.

\section{Method}

Subjects. Eight adult Silver King pigeons served as subjects. All birds had had previous experience with a food-reinforced discrimination between displays of green dots. The subjects were maintained at $80 \%$ of free-feeding weights throughout the current series of experiments.

Apparatus. The test apparatus consisted of a standard operant chamber for pigeons. This enclosed compartment measured $31 \times 35.5$ (floor dimensions) $\times 35.5 \mathrm{~cm}$ (wall height). Three pecking keys were arranged horizontally on the front wall of the chamber, $24 \mathrm{~cm}$ above the floor and spaced $8 \mathrm{~cm}$ apart, center to center. A $6 \times 6 \mathrm{~cm}$ food well was centered on the front wall $9 \mathrm{~cm}$ above the floor, and an electromechanical food hopper supplied mixed grain through this opening. A houselight was attached to the panel containing the pecking keys, $5 \mathrm{~cm}$ above the rim of the center key. The houselight contained a $.1-A$, 28-V lamp, housed within a metal shield that covered the top half of the lamp but left the bottom half exposed. A rectangular translucent cover, which measured $10.5 \times 5 \times 2.5 \mathrm{~cm}$, was placed in the center of the ceiling. Inside this cover were two 6 -W light bulbs, one covered with a red lens and the other with a green lens, which provided either red or green ambient illumination. Auditory stimuli were provided by two Heathkit Model $1 \mathrm{G}-18$ sine-wave tone generators. These tone generators were used to produce a high-pitched tone of $3000 \mathrm{~Hz}$ at $68 \mathrm{~dB}$ and a low-pitched tone of $300 \mathrm{~Hz}$ at $78 \mathrm{~dB}$. Tones were presented through a speaker located on the left side of the front wall. Multistimulus projectors mounted behind the outer pecking keys illuminated the keys with blue or yellow light. Trial presentation and response recording were controlled by an electromechanical system of relays and timers located in a room adjacent to the testing room.

Procedure. The subjects were assigned randomly to either the auditory sample group or the visual sample group, with four subjects in each group. All birds were trained initially on simultaneous matching-to-sample problems. These problems consisted of daily sessions of 48 trials; on each trial, the sample stimulus was presented for $5 \mathrm{sec}$ and the side keys then were illuminated with blue and yellow light. Both the sample stimulus and the side keys remained on until the subject pecked one of the side keys. For the auditory group, a single peck to a blue key during a $3000-\mathrm{Hz}$ tone or to a yellow key during a $300-\mathrm{Hz}$ tone was followed by $2 \mathrm{sec}$ of access to grain. In the visual group, a response to a blue key during red ambient illumination or to a yellow key during green ambient illumination was followed by access to grain. Incorrect responses terminated the trial without reinforcement. A 20 -sec intertrial interval spent in darkness, followed either termination of grain access on correct trials or peck responses on incorrect trials. The blue and yellow colors were presented equally of ten on left and right side keys, in a random sequence.

During the first session of training for each group, the same sample was presented on all trials. The alternative sample then was presented on all trials of the second session. The subjects then were given daily training with sessions in which the same sample appeared on the first 24 trials and the alternative sample 
appeared on the remaining 24 trials. The training continued until each bird achieved three consecutive sessions of $80 \%$ correct responses or better. Upon reaching this criterion, each bird was trained on 48-trial sessions in which the same sample stimulus was presented on 12 successive trials and sample stimuli were alternated after each 12 -trial block. This training continued until each subject once again reached the criterion of $80 \%$ or better accuracy over three successive sessions. The subjects then were placed on sessions in which the sample stimuli were arranged randomly over the 48 trials, with the restrictions that each sample appear on 24 trials and that no more than 3 consecutive trials occur in which the same sample stimulus appeared. Once performance reached the $80 \%$ criterion for 3 successive days with random simultaneous matching to sample, a subject was transferred to $0-\mathrm{sec}$ delayed matching to sample. These sessions were identical to random simultaneous matching, except that the sample stimulus terminated with the illumination of the pecking keys.

When a subject had accomplished three consecutive sessions of 0 -sec delayed matching with $80 \%$ or better accuracy, it began experimental testing on delayed matching to sample. Each session consisted of 48 trials in which the interval between sample stimulus termination and the illumination of the pecking keys varied among values of $0, .5,1$, and 3 sec. Each delay condition was tested 12 times within a session, and the various delays were arranged randomly across trials; each sample stimulus was presented for six trials at each delay. The durations of the sample stimulus were varied among sessions, and values of 1,4 , and $8 \mathrm{sec}$ were tested. Two different random orders of trial presentation were employed, and each sample duration was tested once under each order. Each block of testing consisted of six sessions, and each subject was tested for two blocks of sessions.

\section{Results and Discussion}

Pigeons in the visual group acquired simultaneous matching to sample considerably faster than pigeons in the auditory group. The mean number of total sessions required to complete all of the stages of training was 11.8 for the visual group and 43.2 for the auditory group. The difference between groups was highly significant $[\mathrm{t}(6)=8.5, \mathrm{p}<.001]$, with the scores ranging from 11 to 14 sessions in the visual group and from 33 to 50 sessions in the auditory group. When placed on 0 -sec delayed matching to sample, the visual and auditory groups required 6.8 and 35.8 mean sessions to reach criterion, respectively. The difference between these mean scores was not significant $[t(6)=1.7, p>.05]$. Although scores ranged from 4 to 10 sessions in the visual group, the auditory group showed a bimodal distribution, with two pigeons requiring only 6 and 7 sessions to reach criterion and two others requiring 63 and 67 sessions.

Retention curves that plot percentage of correct responses against delay are presented for visual and auditory groups in Figure 1. The overall level of accuracy is lower in the auditory curves than in the visual curves. In both modalities, accuracy declines as the delay interval is increased and the levels of the curves rise as the sample exposure duration is lengthened.

A mixed analysis of variance revealed significant main effects of sample duration $[F(2,12)=26.7, p<$ $.001]$ and delay interval $[F(3,18)=13.7, p<.001]$. $A$ significant interaction was found of delay interval $X$

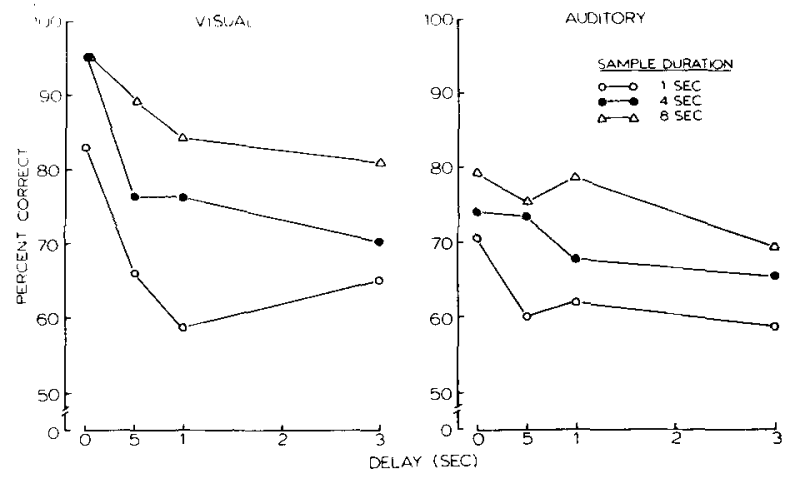

Figure 1. Retention curves for visual and auditory groups tested with varying lengths of exposure to the sample stimulus.

sample duration $[F(6,36)=3.0, p<.05] ;$ this interaction appears to be the result of a slightly more rapid decline in performance over increasing delays for shorter sample durations. Examination of the retention curves suggests that the visual curves fall faster than the auditory curves, and this observation is substantiated by a significant modality $X$ delay interval interaction $[F(3,18)$ $=4.9, \mathrm{p}<.05]$.

The findings of this initial experiment are typical of results from previous studies that used visual sample stimuli presented on a center key (Roberts, 1972; Roberts \& D. S. Grant, 1974). Accuracy in both auditory and visual groups dropped as the delay interval lengthened, and both auditory and visual animals showed increasing accuracy as sample exposure time was raised. The observation that auditory retention curves were flatter than visual retention curves suggests the possibility of different rates of forgetting between modalities. A problem with such a conclusion is that the visual curves begin at a higher level of accuracy than do the auditory curves. It could be argued, for example, that both visual and auditory forgetting curves are controlled by the same exponential rate parameter and that visual curves appear to drop more steeply only because they originate at a higher level of accuracy. We might attempt to overcome this objection by comparing the 1-sec-exposure-duration curve for the visual group with the 8-sec-exposure-duration curve for the auditory group. These curves both begin at around $80 \%$ correct at the 0-sec delay, and it can be seen that the visual curve still falls considerably faster than the auditory curve. This comparison might be objected to, however, on the grounds that exposure duration may affect rate of forgetting. It appears that these data do not allow us to draw any strong conclusions about differences in rates of forgetting between visual and auditory groups.

\section{EXPERIMENT 2}

It has been established that repetition of a sample stimulus improves delayed matching accuracy and that 
performance drops as the interstimulus interval (ISI) between repetitions is made longer (Roberts, 1972; Roberts \& D. S. Grant, 1974). When the intertrial interval (ITI) is varied, performance improves as the ITI becomes longer (Roberts, 1980; Roberts \& Kraemer, 1982). In Experiment 2, the effects of ISI, ITI, and delay were examined in both auditory and visual delayed matching. A $2 \times 3 \times 3 \times 3$ experimental design was used, in which both auditory and visual groups of birds were tested under three levels of ISI $(0,2$, and $6 \mathrm{sec})$, three levels of ITI $(4,16$, and $64 \mathrm{sec})$, and three delay intervals $(0$, 2 , and $5 \mathrm{sec}$ ).

\section{Method}

The same subjects and apparatus used in Experiment 1 were used in Experiment 2. The auditory and visual groups, each containing four pigeons, were tested on daily sessions containing 48 trials. Within a session, auditory birds heard the high tone as a sample stimulus on 24 trials and the low tone as a sample stimulus on the other 24 trials. Similarly, red and green ambient lights were presented to visual subjects as sample stimuli for 24 trials each. For subjects tested in each modality, the two sample stimuli alternated throughout a session in random order. On each trial, the sample stimulus was presented twice for $5 \mathrm{sec}$ each time. The ISI was varied between sessions, so that sample presentations on each trial within a session were separated by 0,2 , or $6 \mathrm{sec}$. In the case of the 0 -sec ISI, the sample stimulus was presented continuously for $10 \mathrm{sec}$. The ITI also was varied between sessions, so that a constant interval of 4,16 , or $64 \mathrm{sec}$ elapsed between trials within each session. The delays occurred in a random sequence, and each sample stimulus was tested eight times at each delay.

Within a block of sessions, each of the nine combinations of ITI and ISI was tested once with each of two orders of trials. A block of sessions then contained 18 days, and subjects were tested for two blocks of sessions. The sequence in which different combinations of ITI $\times$ ISI were tested varied randomly between subjects and between blocks of sessions.

\section{Results and Discussion}

Shown in Figure 2 are visual and auditory retention curves for each length of ITI. As was the case in Experiment 1 , performance tends to drop as the delay becomes longer for both visual and auditory groups. In both modalities, accuracy improves as the ITI is made longer. Analysis of variance showed significant effects of both delay $[\mathrm{F}(2,12)=28.8, \mathrm{p}<.001]$ and $\operatorname{ITI}[\mathrm{F}(2,12)=$ $23.9, \mathrm{p}<.001]$. A significant interaction was found of delay $X$ ITI $[F(4,24)=5.3, p<.05]$. This interaction appears to result from a tendency for the effects of ITI to be more pronounced at 2- and 5-sec delays than at the 0 -sec delay. The interaction appears to be more pronounced in the visual group than in the auditory group, but this effect does not interact significantly with modality.

The main effect of ISI did not approach significance $(F<1.0)$, and hence the data presented in Figure 2 have been collapsed over the ISI variable. Two other significant main effects found in these data are those of modality $[F(1,6)=15.3, \mathrm{p}<.05]$ and trial blocks $[F(1,6)=12.8, p<.05]$. From Figure 2, it can be seen that the auditory group performed at a lower level than

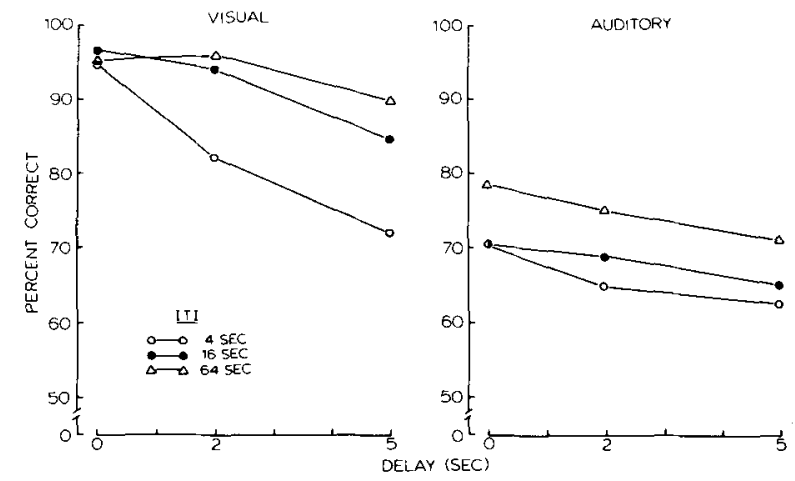

Figure 2. Retention curves for visual and auditory groups tested at varying lengths of the intertrial interval (ITI).

the visual group. Overall, the visual group was correct on $89.4 \%$ of their trials, and the auditory group was correct on $69.8 \%$ of their trials. The significant effect of trial blocks reflected improvement from $77.5 \%$ on Block 1 to $82.0 \%$ on Block 2. The visual and auditory retention curves did not drop at significantly different rates, since the modality $X$ delay interval interaction was not significant $[F(2,12)=3.27, p>.05]$.

Roberts and Kraemer (1982) reported that, in visual delayed matching with identical sample and comparison stimuli presented on keys, percentage of correct choices increased as a linear function of the log of the ratio of the intertrial interval (I) over the delay (D). In Figure 3, the data from Experiment 2 are plotted as a function of the I/D ratio on a $\log$ scale. The relationship found by Roberts and Kraemer seems to hold up well with visual and auditory symbolic delayed matching, as linear curves fit both sets of data fairly well. The correlation between $\log$ I/D and percentage of correct responses

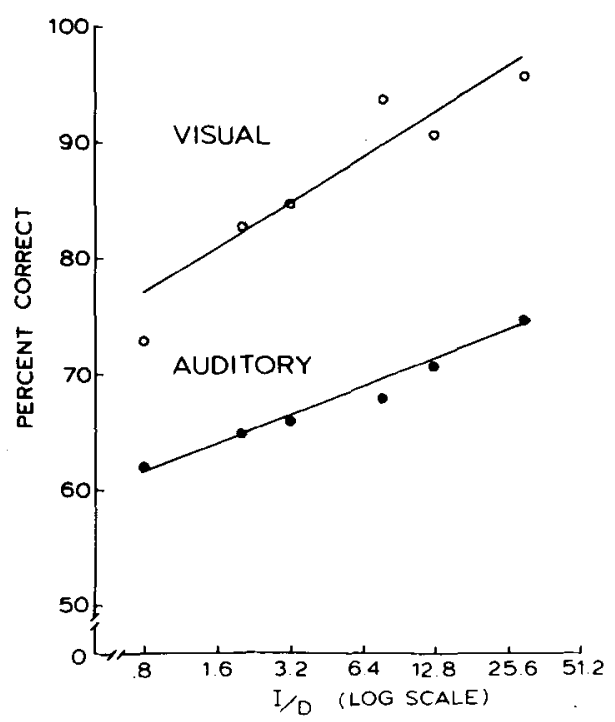

Figure 3. Percentage of correct choices plotted as a function of the I/D ratio (log scale) for visual and auditory groups. 
was $r=.90$ for the visual data and $r=.99$ for the auditory data.

Animals were able to match more accurately as the ITI was increased, and accuracy improved as a linear function of the $\log$ I/D ratio, both with auditory and visual sample stimuli. These findings are in clear agreement with previous studies, in which spaced trials facilitated performance and the sample stimuli were projected on a center key (Roberts, 1980; Roberts \& Kraemer, 1982). Although the auditory group was significantly less accurate than the visual group, the retention curves indicated little difference in rates of forgetting between groups. The failure to find an effect of ISI was surprising, since clear effects of this variable had been observed in previous research (Roberts, 1972; Roberts \& D. S. Grant, 1974). The failure to find an ISI effect did not distinguish between modalities, however, since it was common to both the visual and auditory groups.

\section{EXPERIMENT 3}

In Experiment 3, the question of retroactive interference (RI) effects in visual and auditory delayed matching was addressed. It is well established that animals trained to perform visual delayed matching with the delay spent in darkness will show substantial loss of accuracy on trials with illumination introduced during the delay (D'Amato \& O'Neill, 1971; D. S. Grant \& Roberts, 1976; Roberts \& D. S. Grant, 1978). On the other hand, auditory stimulation introduced during the delay in visual delayed matching experiments with pigeons and monkeys has little or no effect on performance (Thompson, Van Hemel, Winston, \& Pappas, 1983; Wilkie, Summers, \& Spetch, 1981 ; Worsham \& D'Amato, 1973). When the task is auditory delayed matching and the subject is a dolphin, interpolated sound stimulation does produce RI (Herman, 1975).

Although the mechanism responsible for light-induced RI has not yet been specified, Roberts and D. S. Grant (1978) have argued that it arises from forgetting or loss of information. One model of STM that can be enter. tained is that visual sample stimuli establish traces that maintain some of the visual properties of the sample stimulus. The presentation of interpolated stimulation in the same modality, white light, acts to degrade the visual trace and hence causes RI. Stimulation presented in a different modality, auditory stimulation, will have little effect on a visual trace. The suggestion here is that RI is modality specific and that only stimulation presented in the same modality as the sample stimulus will produce RI. Some evidence in support of modality specificity can be found in the human STM literature (Broadbent, Vines, \& Broadbent, 1978; Kroll, Parks, Parkinson, Bieber, \& Johnson, 1970; Massaro \& Kahn, 1973; Proctor, 1978). In these experiments, subjects were required to remember visually or aurally presented items, with interfering information presented during the retention interval in either the same or the opposite modality. It was found that retention was lower when the interfering and to-be-remembered materials were presented in the same modality than when they were presented in different modalities.

The notion of modality specificity assumes that STM in pigeon delayed matching is at least partially retrospective. As an alternative model, the prospective position suggests that sample stimuli rapidly become coded into response instructions that do not have modality-unique characteristics. In this case, light may produce RI because it introduces stimulation that competes with the maintenance or rehearsal of response instructions. Noise introduced during a delay may cause little RI because it is not as demanding or salient a form of stimulation.

Experiment 3 may be seen as one test of these two models of STM and RI. The fact that light, but not sound, produces strong RI effects in visual delayed matching can be explained by both models. In Experiment 3 , both visual and auditory groups were subjected to light and noise as interpolated stimulation between sample and comparison stimuli. The retrospective and prospective models outlined now would seem to make different predictions. If visual and auditory stimuli established modality-unique traces, and each type of trace was degraded only by interpolated stimulation presented in its own modality, then we would expect an interaction between the effects of sample and interpolated stimuli. That is, interpolated light should interfere only with visual delayed matching, and interpolated noise should interfere only with auditory delayed matching. On the other hand, if visual and auditory sample stimuli established response instructions with common properties, we would expect both groups to show strong RI effects with interpolated light but not with interpolated noise. If light interferes with the maintenance of response instructions, it should do so to an equivalent degree in both visual and auditory groups.

\section{Method}

The subjects used in Experiments 1 and 2 were tested again. Subjects in the appropriate groups were tested daily in 48-trial sessions of visual or auditory delayed matching, with the sample stimulus presented for $5 \mathrm{sec}$ on each trial and with the conditions in effect during the delay interval varied between trials. Within each session, 12 trials were devoted to each of four possible post-sample-stimulus events. On three types of trials, a 5 -sec delay interval occurred between sample stimulus termination and illumination of the sidekeys. During this 5-sec delay, the chamber was darkened in the control condition, the houselight was turned on in the interpolated illumination condition, or white noise was played into the darkened chamber in the interpolated noise condition. The houselight was presented at full intensity on interpolated illumination triais, and white noise at $77 \mathrm{~dB}$ was played on interpolated noise trials. The fourth type of trial was a $0-\mathrm{sec}$ delay condition, in which the sidekeys were illuminated at the offset of the sample stimulus. The four conditions were presented in random orders within sessions, with the frequency of presentation of each sample stimulus and the left-right positions of the correct comparison stimulus balanced within each delay condition. Two different 
random orders of trial presentation were used, and these orders alternated regularly over sessions. Twelve sessions constituted a block of trials, and all pigeons were tested for two blocks of trials.

\section{Results and Discussion}

The percentages of correct responses are shown for each delay condition within visual and auditory groups in Table 1. Initial inspection of the table suggests that, as in the preceding two experiments, delayed matching to visual sample stimuli was superior to delayed matching to auditory sample stimuli. Both visual and auditory groups show comparable drops in accuracy from the 0 -sec condition to the 5-sec delay control condition. The data in just the first two columns of Table 1 were subjected to a modality $X$ delay analysis of variance. Significant effects were found for both modality $[F(1,6)=$ $29.3, \mathrm{p}<.01]$ and delay $[\mathrm{F}(1,6)=30.8, \mathrm{p}<.01]$ but not for the modality $X$ delay interaction $(F<1.0)$.

In order to assess the influence of delay stimulation on performance, accuracy on trials with interpolated light and interpolated noise was compared with accuracy on the 5-sec delay control trials separately for the visual and auditory groups. In the visual group, accuracy on interpolated light trials was significantly below that of the control condition $[\mathrm{t}(3)=8.2, \mathrm{p}<.01]$. Although the effect of interpolated noise on visual matching appears to be negligible, the noise condition was significantly lower than the control $[\mathrm{t}(3)=5.9, \mathrm{p}<.01]$. A further direct comparison between the interpolated light and noise conditions in the visual group revealed a significant difference $[t(3)=6.6, p<.01]$. A similar pattern of effects appeared within the auditory group. Interpolated light led to significantly lower accuracy than was found in the control condition [ $t(3)=5.81, \mathrm{p}<.01]$, but interpolated noise did not significantly affect performance relative to the control $[\mathrm{t}(3)=1.9, \mathrm{p}>.05]$. The difference between the interpolated light and noise conditions also was significant $[\mathrm{t}(3)=3.7, \mathrm{p}<.05]$.

The data were examined for practice effects. In the auditory group, overall accuracy improved from $64.9 \%$ to $66.4 \%$ over Trial Blocks 1 and 2, respectively, but the difference was not significant $[\mathrm{t}(3)=1.2, \mathrm{p}>.05]$. For visual animals, accuracy improved from $79.6 \%$ in Block 1 to $83.7 \%$ in Block 2, and this increase was significant $[t(3)=2.6, p<.05]$.

The finding of major interest in Experiment 3 concerns the observation that interpolated light and noise had essentially parallel effects on the accuracy of delayed matching to visual and auditory sample stimuli.
The notion of modality specificity led to the prediction that interpolated light would interfere with visual delayed matching but not with auditory delayed matching, and that interpolated noise would interfere with auditory delayed matching but not with visual delayed matching. Contrary to this prediction, interpolated light substantially reduced the accuracy of both visual and auditory matching, whereas interpolated noise had little or no effect within both modalities.

\section{EXPERIMENTS 4A AND 4B}

The results from the visual group in Experiment 3 are in good agreement with the findings of other STM experiments that have introduced interpolated light and noise stimulation; light produced a strong RI effect, and noise had little effect (Thompson et al., 1983; Wilkie et al., 1981; Worsham \& D'Amato, 1973). Common to all of these experiments was the use of only one level of sound intensity. It is possible that the levels used did not produce strong enough sensory effects to interfere with memory and that more intense sound stimulation would yield a substantial RI effect. Interference with visual delayed matching increases as a direct function of the intensity of interpolated houselight illumination (D. S. Grant \& Roberts, 1976). The levels of interpolated sound intensity used thus far might be psychologically equivalent only to dim intensities of houselight.

The design of Experiments $4 \mathrm{~A}$ and $4 \mathrm{~B}$ was similar to that of Experiment 3, except that the intensity of interpolated stimulation was varied over three levels. Both visual and auditory delayed matching were examined at three intensities of interpolated white noise, all three of which were more intense than the level used in Experiment 3. As a parallel manipulation, the intensity of interpolated white light was varied over three levels; the full intensity condition used in Experiment 3 was the highest level, and two weaker degrees of illumination were added. Experiments $4 \mathrm{~A}$ and $4 \mathrm{~B}$ differed only in that, in an attempt to raise the level of accuracy in the auditory group, Experiment $4 \mathrm{~B}$ used a shorter delay than Experiment $4 \mathrm{~A}$ for that group.

One possible outcome of this experiment was that interference effects might be found with more intense interpolated noise with both visual and auditory delayed matching. On the other hand, interpolated noise might continue to have no effect on delayed matching in either modality, even at stronger levels of intensity. Both of

Table 1

Percent Correct Responses in Each Delay Condition of Experiment 3 for Visual and Auditory Groups

\begin{tabular}{|c|c|c|c|c|}
\hline \multicolumn{5}{|c|}{ Delay Condition } \\
\hline $\begin{array}{l}\text { Auditory } \\
\text { Visual }\end{array}$ & $\begin{array}{l}78.2 \\
95.2\end{array}$ & $\begin{array}{l}67.1 \\
85.7\end{array}$ & $\begin{array}{l}51.6 \\
62.7\end{array}$ & $\begin{array}{l}65.9 \\
83.1\end{array}$ \\
\hline
\end{tabular}


these findings would be in keeping with the notion of prospective memory. The possibility existed, however, that some findings in agreement with retrospective memory and modality specificity would appear at higher levels of interpolated sound; that is, intense interpolated sound could interfere with memory of auditory sample stimuli but not with memory of visual sample stimuli.

\begin{abstract}
Method
The same groups of subjects tested in the previous cxperiments were tested again, with ambient tones and colored lights as sample stimuli for the auditory and visual groups, respectively. As in Experiment 3, each session consisted of 48 trials, with the sample stimulus presented for $5 \mathrm{sec}$ on each trial; 12 trials were devoted to each of the conditions of 0 -sec delay, 5-sec delay in darkness (control), 5-sec delay with houselight, and $5-\mathrm{sec}$ delay in darkness with white noise. Between daily sessions, the levels of interpolated housclight and white noise were varied. The houselight was varied in intensity by placing cither a 300 - or a $600-\Omega$ resistor in series with the houselight. These levels of illumination were referred to as low and medium; a high level of illumination was achicved by using the houselight at full intensity. A Tektronix J16 digital photometer was placed immediately adjacent to the houselight and gave readings of 4,30 , and $7500 \mathrm{~cd} / \mathrm{m}^{2}$ at the low, medium, and high settings, respectively. In the case of interpolated white noise, low, medium, and high intensities corresponded to sounds of 83,88 , and $93 \mathrm{~dB}$. Each of the nine combinations of each level of houselight intensity with each level of white noise intensity was tested once with each of two random orders of trials. An entire block of testing occupied 18 days. The first block of sessions was labeled Experiment $4 \mathrm{~A}$. In a second block of sessions, exactly the same procedures were followed, except that the delay was reduced from 5 to $2 \mathrm{sec}$ for the auditory group. The second block of sessions was labeled Experiment 4B. The orders in which the different combinations of light and noise intensity were tested varied randomly between birds and between blocks of sessions.
\end{abstract}

\section{Results and Discussion}

Experiment 4A. Within the visual group, the mean percentage of correct responses was 94.2 at the 0 -sec delay and decreased to 90.8 at the 5-sec delay control condition. The corresponding percentages for the auditory group were 84.8 at the 0 -sec delay and 67.4 at the 5-sec delay. Statistical analysis showed significant effects of both modality $[F(1,6)=16.4, p<.01]$ and delay $[F(1,6)=10.12, p<.05]$. The means suggest a greater drop in accuracy from 0 to $5 \mathrm{sec}$ for the auditory group than for the visual group, and the modality $X$ delay interaction approached, but did not reach, significance $[F(1,6)=4.56, p>.05]$

The effects of interpolated light and noise on performance are shown relative to the control level of accuracy in Figure 4. For both the visual data in the left panel and the auditory data in the right panel, percentage of correct responses has been plotted as a function of low $(\mathrm{L})$, medium (M), and high $(\mathrm{H})$ levels of interpolated houselight and white noise. In general, similar effects of interpolated stimulation are found with both visual and auditory delayed matching; interpolated noise led to only a slight reduction in accuracy below the control level, but interpolated light produced more substantial

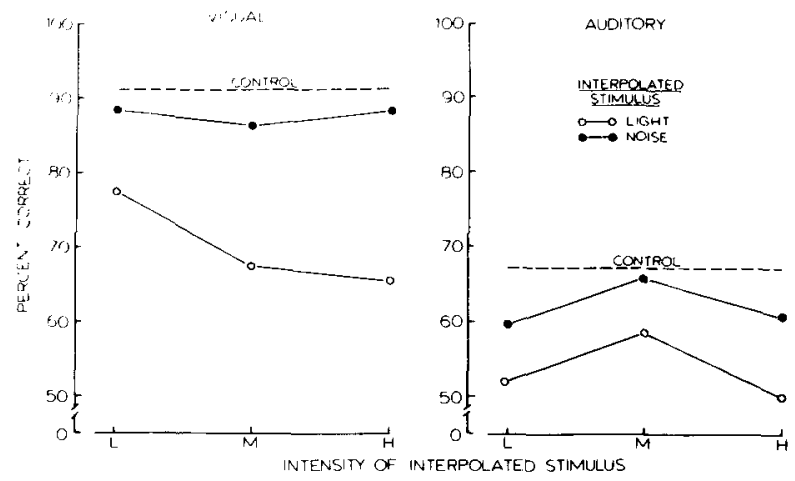

Figure 4. Percentage of correct choices plotted as a function of low (L), medium (M), or high $(\mathrm{H})$ intensities of interpolated houselight or white noise (Experiment 4A).

losses in accuracy. Increasing the intensity of the white noise did not lead to a systematic increase in interference in either group. When the intensity of the houselight was increased, performance dropped consistently for the visual group but not for the auditory group.

Performance in the control condition and the three levels of light or noise intensity was compared in oneway analyses of variance. No significant effect was found among the control condition and the noise conditions in either the visual group $(F<1.0)$ or the auditory group $[\mathrm{F}(3,9)=1.4, \mathrm{p}>.05]$. Performance during interpolated light was significantly impaired relative to control trials in both the visual group $[F(3,9)=7.2$, $p<.01]$ and the auditory group $[F(3,9)=4.9, p<.05]$. Dunnett tests were used to compare performance at the various light intensities with the control condition $(\alpha=.05)$. For the visual group, both medium- and highintensity light conditions significantly disrupted performance relative to the control trials, whereas a lowintensity light did not. In the case of auditory delayed matching, performance at low and high light intensities was significantly inferior to control performance, but accuracy during medium-intensity light did not differ significantly from that of the control. Finally, comparisons made across just the low, medium, and high levels of stimulus intensity showed no significant effects of either light or noise intensity in either group.

Experiment 4B. Performance generally was higher in Experiment 4B than in Experiment 4A, and this improvement was more marked in the auditory group than in the visual group. In the visual group, the mean percentages of correct responses were 95.4 at the 0 -sec delay and 92.6 at the 5-sec delay control condition. For the auditory group, accuracy dropped from $90.8 \%$ correct at the 0 -sec delay to $81.2 \%$ correct at the $2-\mathrm{sec}$ delay control condition. Since the control delays used varied between visual and auditory groups, the scores obtained at these delays are not strictly comparable in an analysis of variance. However, a modality $X$ delay analysis was carried out and may be considered a conservative test of the hypothesis that the auditory reten- 
tion curve fell faster than the visual retention curve. Significant effects were found for modality $[F(1,6)=$ $10.0, p<.05]$, delay $[F(1,6)=29.9, p<.01]$, and the modality $X$ delay interaction $[F(1,6)=9.0, p<.05]$.

In Figure 5 , the percentages of correct responses are plotted against $\mathrm{L}, \mathrm{M}$, and $\mathrm{H}$ levels of houselight and white noise intensity for trials carried out in Experiment 4B. These results fairly closely mirror the results of Experiment 4A. Once again, interpolated noise appears to exert only a minor influence on performance, whereas interpolated light has a major effect. With the increased level of overall accuracy found in the auditory group, degree of interference produced by light in this group is greater than that found in Experiment 4A. This observation suggests that the degree of interference caused by interpolated light in the auditory group in Experiment $4 \mathrm{~A}$ may have been attenuated by a floor effect. As in Experiment $4 \mathbf{A}$, the only suggestion of a decline in accuracy with increasing intensity of an interpolated stimulus is found for houselight intensity in the visual group.

No significant differences were found between the control condition and the three levels of noise intensity in either the visual group $(F<1.0)$ or the auditory group $[F(3,9)=1.5, p>.05]$. Light interpolated during the delay produced a significant decrement in performance relative to the control condition in both the visual group $[F(3,9)=32.1, p<.001]$ and the auditory group $[F(3,9)=11.0, p<.01]$. Dunnett tests showed that performance at all levels of light intensity was significantly disrupted relative to control trials for both visual and auditory groups. In addition, it was found that performance declined significantly as light intensity increased in the visual group $[F(2,6)=22.5, p<.01]$. No other significant effects of stimulus intensity values were found in either group.

The results of Experiments $4 \mathrm{~A}$ and $4 \mathrm{~B}$ confirm the findings of Experiment 3 . Houselight interpolated between sample and comparison stimuli strongly interfered with the accuracy of delayed matching, but interpolated white noise had little effect. The differential

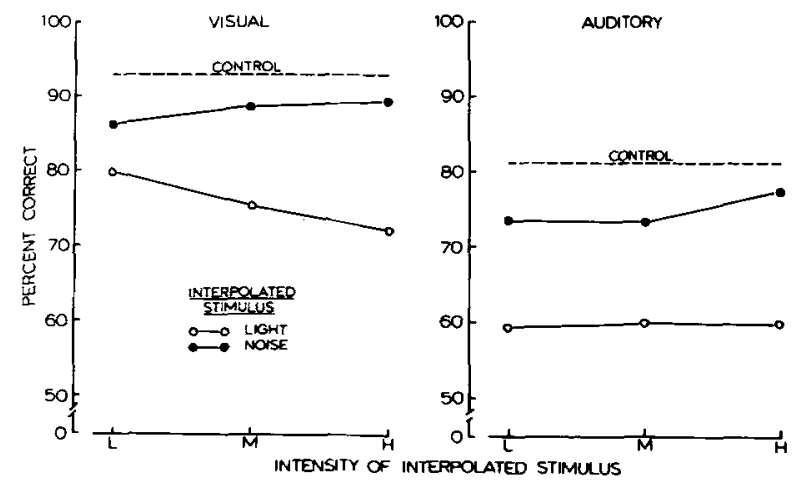

Figure 5. Percentage of correct choices plotted as a function of low (L), medium (M), or high (H) intensities of interpolated houselight or white noise (Experiment 4B). effects of these two sources of interpolated stimulation do not interact with the modality of the sample stimulus. It was suggested that the failure to find RI caused by interpolated noise in Experiment 3 might have arisen from the failure to use a sufficiently intense noise. The results of Experiments $4 \mathrm{~A}$ and $4 \mathrm{~B}$ argue against that suggestion, since no significant RI effect was found at any of the three noise levels used, each of which was higher in intensity than the noise level used in Experiment 3. Furthermore, there was no suggestion that performance was dropping as the intensity of interpolated white noise was increased.

\section{GENERAL DISCUSSION}

The general purpose of these experiments was to compare STM in pigeons for visual and auditory stimuli. Separate groups of birds trained to perform visual and auditory delayed matching were compared by determining how a number of different variables affected their performance. Two different approaches or conceptualizations of animal STM suggest possible different outcomes to these experiments. One possibility is that pigeons, after being presented with visual or auditory sample stimuli, retain memories that differ in terms of the preservation of visual or auditory components. This conceptualization clearly suggests that STM is retrospective in nature. If memories of visual and auditory stimuli differed in this way, we might expect to find quantitative and/or qualitative differences in retention of these stimuli. The alternative position is that visual and auditory stimuli are coded immediately into response instructions regarding the comparison stimuli (D. S. Grant, 1981; Honig \& Thompson, 1982; Roitblat, 1980 ). When the comparison stimuli are presented, the subject only needs to execute the remembered response instruction. If pigeons are using instructional memory, the codes remembered during the delay interval should be identical for visual and auditory birds, since both groups will have had the same comparison stimuli. The prospective memory position suggests that we should find little in the way of differences between visual and auditory groups, either in terms of rates of forgetting or in the effects of different variables on performance.

Given the apparent dominance of the visual modality in pigeons, we might expect pigeons to show slower forgetting of visual memories than of auditory memories. On the other hand, there is evidence to indicate better retention of aurally presented information than of visually presented information in both people (Conrad \& Hull, 1968; Murdock, 1968; Murdock \& Walker, 1969; Watkins et al., 1974) and rats (Cohen et al., 1984; Wallace et al., 1978). Unfortunately, the data reported in these experiments do not allow us to draw any firm conclusions about the memorability of visual and auditory stimuli in pigeons. One problem is created by the fact that the auditory group generally showed 
lower accuracy than the visual group. It may be that the auditory stimuli were more difficult for birds to discriminate than were the visual stimuli. As a consequence of this difference in accuracy, auditory retention curves were lower than visual retention curves at the 0 -sec delay, and problems concerned with the equivalency of units of forgetting between visual and auditory groups are raised. Even when absolute rates of forgetting are compared between auditory and visual groups, the results are not consistent. The findings of Experiment 1 suggested flatter retention functions for the auditory group than for the visual group. However, Experiments 2 and 3 indicated little difference in the slopes of retention curves, and evidence of superior retention of visual stimuli was found in Experiments $4 \mathrm{~A}$ and $4 \mathrm{~B}$. It is possible that a differential practice effect in favor of retention of visual stimuli was occurring throughout these experiments. In any case, the relative retention of visual and auditory cues will be established only by further research.

Other aspects of these experiments do offer evidence in favor of the prospective memory position. With regard to possible differential effects of variables on visual and auditory delayed matching, Experiments 1 and 2 showed that both groups were similarly affected by manipulations of sample exposure time and the ITI. That is, accuracy improved as both sample exposure duration and ITI were increased in both visual and auditory groups, and the extent of improvement was essentially equivalent between groups, since there was no interaction of either variable with modality. It was found also that both the visual and auditory groups showed a linear relationship between the percentage of correct responses and the $\log$ I/D ratio. In Experiments 3, 4A, and 4B, similar effects of interpolated houselight and white noise were found in visual and auditory groups. Although interpolated white noise generally did not produce a significant RI effect in these experiments, it consistently produced performance slightly lower than control accuracy across experiments. This observation suggests that noise may have had a mild disrupting effect upon retention, and this observation would be in keeping with recent findings of Thompson et al. (1983) with a delayed discrimination procedure. Only interpolated houselight produced strong RI effects, and this was equally true for both visual and auditory delayed matching. In summary, light interfered equally strongly with visual and auditory delayed matching, and noise had only slight effects on retention of stimuli presented to each modality. This finding offers no support for the notion of modality specificity in pigeon STM and is in contrast with findings from human STM experiments, in which interfering information presented in the same modality as the to-be-remembered information often produces more forgetting than does interfering information presented in a different modality (Broadbent et al., 1978; Kroll et al., 1970; Massaro \& Kahn, 1973; Proctor, 1978). These findings suggest that light-induced RI does not arise from a specific degrading effect of houselight on a visual sensory trace. A more likely possibility is that houselight interferes with the rehearsal of response instructions. Common response instructions engendered by visual or auditory sample stimuli then would be equally disrupted by houselight.

Two differences between visual and auditory delayed matching did appear. The first was a tendency for accuracy to drop as a function of increasing intensity of interpolated light in the visual group but not in the auditory group (Experiments $4 \mathrm{~A}$ and $4 \mathrm{~B}$ ). The meaning of this difference is not clear from the point of view of a prospective model, but the difference does agree with a retrospective model in which it is held that a visual trace is more sensitive to light intensity than an auditory trace. This finding is admittedly anomalous within the general context of agreement between visual and auditory effects. The other difference between the two modality groups is the higher level of accuracy found in the visual group than in the auditory group. It may be that pigeons are not biologically prepared to discriminate auditory stimuli as well as visual stimuli. On the other hand, it may be that the frequencies of the tones used as sample stimuli did not give rise to sufficiently distinct sensations to permit better discrimination. The use of tones with more disparate frequencies might yield better discrimination, or the use of auditory stimuli varying along other dimensions might give rise to higher accuracy. Whatever the reason for poorer discrimination of auditory stimuli, a prospective memory model suggests that lower accuracy with auditory samples resulted from the frequent formation of erroneous response codes. Failure to discriminate well between $300-$ and $3000-\mathrm{Hz}$ tones would often lead a pigeon to form an inappropriate code, such as "peck yellow" when "peck blue" was correct and "peck blue" when "peck yellow" was correct.

\section{RAPERENCES}

BLough, D. S. (1959). Delayed matching in the pigeon. Journal of the Experimental Analysis of Behavior, 2, 151-160.

Broadbent, D., Vines, R., \& Broadbent, M. (1978). Recency effects in memory, as a function of modality of intervening events. Psychological Research, 40, 5-13.

Cohen, J. S., Escott, M., \& Riccinadi, P. (1984). The role of reinforcement symmetry and stimulus modality in successive delayed matching to sample in the rat. Canadian Journal of Psychology, 38, 63-79.

Conran, R., \& Hull, A. J. (1968). Input modality and the serial position curve in short-term memory. Psychonomic Science, 10, 135-136.

Cooley, R. K., \& McNulty, J. A. (1967). Recall of individual CCC trigrams over short intervals of time as a function of mode of presentation. Psychonomic Science, 9, 543-544.

D'Amato, M. R., \& O'NEtLL, W. (1971). Effect of delay-interval illumination on matching behavior in the capuchin monkey. Journal of the Experimental Analysis of Behavior, 15, 327-333.

Grant, D. S. (1981). Short-term memory in the pigeon. In N. E. Spear \& R. R. Miller (Eds.), Information processing in animals: Memory mechanisms. Hillsdale, NJ: Erlbaum. 
Grant, D. S. (1982a). Prospective versus retrospective coding of samples of stimuli, responses, and reinforcers in delayed matching with pigeons. Learning and Motivation, 13, 265-280.

Grant, D. S. (1982b). Samples of stimuli, responses, and reinforcers: Effect of incongruent sample type, serial position, and mode of presentation. Animal Learning Behavior, 10, 7-14.

Grant, D. S., \& Roberts, W. A. (1976). Sources of retroactive inhibition in pigeon short-term memory. Journal of Experimental Psychology: Animal Behavior Processes, 2, 1-16.

Grant, K. W., \& McCormack, P. D. (1969). Auditory and visual short-term memory with successive syllable presentation in both modalities. Psychonomic Science, 17, 341-342.

Herman, L. M. (1975). Interference and auditory short-term memory in the bottlenosed dolphin. Animal Learning Behavior, 3, 43-48.

Herman, L. M. (1980). Cognitive characteristics of dolphins. In L. M. Herman (Ed.), Cetacean behavior: Mechanisms and functions. New York: Wiley-Interscience.

Herman, L. M., \& Gordon, J. A. (1974). Auditory delayed matching in the bottlenose dolphin. Journal of the Experimental Analysis of Behavior, 21, 19-26.

Herman, L. M., \& Thompson, R. K. R. (1982). Symbolic, identity, and probe delayed matching of sounds by the bottlenosed dolphin. Animal Learning \& Behavior, 10, 22-24.

Honig, W. K., \& Thompson, R. K. R. (1982). Retrospective and prospective processing in animal working memory. In G. $H$. Bower (Ed.), The psychology of learning and motivation (Vol. 16). New York: Academic Press.

Kroli, N. E. A., Parks, T., Parkinson, S. R., Bieger, S. L., \& Johnson, A. L. (1970). Short-term memory while shadowing: Recall of visually and of aurally presented letters. Journal of Experimental Psychology, 85, 220-224.

Maki, W. S., Moe, J. C., \& Bienley, C. M. (1977). Short-term memory for stimuli, responses, and reinforcers. Journal of Experimental Psychology: Animal Behavior Processes, 3, 156-177.

MAssaro, D. W., \& KAHN, B. J. (1973). Effects of central processing on auditory recognition. Journal of Experimental Psychology, 97, 51-58.

MURDOCK, B. B. (1968). Modality effects in short-term memory: Storage or retrieval? Journal of Experimental Psychology, 77, 79-86.

Murdock, B. B., \& W Alker, K. D. (1969). Modality effects in free recall. Journal of Verbal Learning and Verbal Behavior, 8 , 665-676.

Nelson, K. R., \& Wasserman, E. A. (1978). Temporal factors influencing the pigeon's successive matching-to-sample performance: Sample duration, intertrial interval, and retention interval. Journal of the Experimental Analysis of Behavior, 30, 153-162.

Peterson, G. B., Wheeler, R. L., \& Armstrong, C. D. (1978). Expectancies as mediators in the differential-reward conditional discrimination performance of pigeons. Animal Learning \& $B e$ havior, 6, 279-285.
Peterson, L. R., \& Peterson, M. J. (1959). Short-term retention of individual verbal items. Journal of Experimental Psychology, 58, 193-198.

Proctor, R. W. (1978). Attention and modality-specific interference in visual short-term memory. Journal of Experimental Psychology: Human Learning and Memory, 4, 239-245.

Roвerts, W. A. (1972). Short-term memory in the pigeon: Effects of repetition and spacing. Journal of Experimental Psychology, 94, 74-83.

RoBents, W. A. (1980). Distribution of trials and intertrial retention in delayed matching to sample with pigeons. Journal of $E x$ perimental Psychology: Animal Behavior Processes, 6, 217-237.

Roberts, W. A., \& Grant, D. S. (1974). Short-term memory in the pigeon with presentation time precisely controlled. Learning and Motivation, 5, 393-408.

Roberts, W. A., \& Grant, D. S. (1978). An analysis of lightinduced retroactive inhibition in pigeon short-term memory. Journal of Experimental Psychology: Animal Behavior Processes, 4, 219-236.

Roberts, W. A., \& Kraemer, P. J. (1982). Some observations of the effects of intertrial interval and delay on delayed matching to sample in pigeons. Journal of Experimental Psychology: Animal Behavior Processes, 8, 342-353.

Roitblat, H. L. (1980). Codes and coding processes in pigeon short-term memory. Animal Learning \& Behavior, 8, 341-351.

Spetch, M. L., \& Wilkie, D. M. (1983). Subjective shortening: A model of pigeons' memory for event duration. Journal of Experimental Psychology: Animal Behavior Processes, 9, 12-30.

Thompson, R. K. R., \& Herman, L. M. (1977). Memory for lists of sounds by the bottlenosed dolphin: Convergence of memory processes with humans? Science, 195, 501-503.

Thompson, R. K. R., Van Hemel, P. E., Winston, K. M., \& Pappas, N. (1983). Modality specific interference with overt mediation in a delayed discrimination task by pigeons. Learning and Motivation, 14, 271-303.

Wallace, J., Steinert, P. A., Scobie, S. R., \& Spear, N. E. (1978). Stimulus modality and short-term memory in rats. Animal Learning \& Behavior, 8, 10-16.

Watkins, M. J., Watkins, O. G., \& Crowder, R. G. (1974). The modality effect in free and serial recall as a function of phonological similarity. Journal of Verbal Learning and Verbal Behavior, 13, 430-447.

Wilkie, D. M., Summens, R. J., \& SPetch, M. L. (1981). Effect of delay-interval on delayed symbolic matching to sample in the pigeon. Journal of the Experimental Analysis of Behavior, 35, 153-160.

Worsham, R. W., \& D'Amato, M. R. (1973). Ambient light, white noise, and monkey vocalization as sources of interference in visual short-term memory of monkeys. Journal of Experimental Psychology, 99, 99-105.

(Manuscript received October 28, 1984; revision accepted for publication February 22, 1984.) 